doi:10.13108/2020-12-4-41

\title{
ON COVERING MAPPINGS IN GENERALIZED METRIC SPACES IN STUDYING IMPLICIT DIFFERENTIAL EQUATIONS
}

\author{
E.S. ZHUKOVSKIY, W. MERCHELA
}

\begin{abstract}
Let on a set $X \neq \emptyset$ a metric $\rho: X \times X \rightarrow[0, \infty]$ be defined, while on $Y \neq \emptyset$ a distance $d: Y \times Y \rightarrow[0, \infty]$, be given, which satisfies only the identity axiom. We define the notion of covering and of Lipschitz property for the mappings $X \rightarrow Y$. We formulate conditions ensuring the existence of solutions $x \in X$ to equations of form $F(x, x)=y$, $y \in Y$, with a mapping $F: X \times X \rightarrow Y$, being covering in one variable and Lipschitz in the other. These conditions are employed for studying the solvability of a functional equation with a deviation variable and of a Cauchy problem for an implicit differential equation. In order to do this, on the space $S$ of Lebesgue measurable functions $z:[0,1] \rightarrow \mathbb{R}$ we define the distance

$$
d\left(z_{1}, z_{2}\right)=\text { vrai } \sup _{t \in[0,1]} \theta\left(z_{1}(t), z_{2}(t)\right), \quad z_{1}, z_{2} \in S,
$$

where each continuous function $\theta: \mathbb{R} \times \mathbb{R} \rightarrow[0, \infty)$ satisfies $\theta\left(z_{1}, z_{2}\right)=0$ if and only if $z_{1}=z_{2}$.
\end{abstract}

Keywords: covering mapping, metric space, functional equation with a deviating variable, ordinary differential equation, existence of solution.

Mathematics Subject Classification: 34A09; 47J05; 54E40

\section{INTRODUCTION}

The results on operator equations with mappings acting in metric spaces are widely used for studying various functional equations. In particular, the results on covering mappings of metric spaces allowed one to consider some classes of integral equations, see [1], implicit differential equations, see [2], for which fixed point theorems were not applicable. For implicit differential equations, by such methods, there were obtained existence conditions, estimates, conditions ensuring continuous dependence on parameters for solutions to Cauchy problems, see [3], to boundary value problems, see [4], control problems, see [5], [6].

In recent studies [7], 8], the results on covering mappings were generalized for the spaces, in which classical properties of metrics were weakened. In works [9], [10], the notion of the covering set was extended to mappings acting from a metric space into a set equipped with a distance obeying just identity axiom and by means of this set, solvability conditions of operator equations were obtained. In this work, we extend this result for the case, when the metric and distance can take infinite value. Such generalization gave an opportunity to apply the results on an operator equation to studying a functional equation with a deviating argument in the space of Lebesgue measurable functions as well as for studying an implicit differential equation.

E.S. Zhukovskiy, W. Merchela, On covering mappings in Generalized metric SPaCes In StudyING IMPLICIT DIFFERENTIAL EQUATIONS.

(C) Zhukovskiy E.S., Merchela W. 2020.

The research is financially supported by Russian Science Foundation (project no. 20-04-60524_virusy). Theorem 3.1, Proposition 4 and Corollary 2 are obtained by the first author in Trapeznikov Institute of Control Sciences under the support of Russian Science Foundation (project no. 20-11-20131).

Submitted March 23, 2020. 
The paper is organized as follows. In Section 2 we provide needed facts on spaces with distance, define weakened properties of closedness, covering and Lipschitz property for the mappings acting from a space with an $\infty$-metric into the space with a distance and we also formulate Theorem 2.1 of existence of a solution to an operator equation. In Section 3 we define the distance in the space of Lebesgue measurable functions and in this space we study the covering set and Lipschitz property set for Nemytskii operator. Then we apply the obtained results to studying a functional equation with a deviating argument. In Section 4, by similar methods, we obtained the solvability conditions of a Cauchy problem for an implicit differential equation.

\section{MAIN NOTIONS}

We denote $\mathbb{R}_{+}=[0,+\infty), \overline{\mathbb{R}}_{+}=[0,+\infty]$. We suppose that we are given a space $X=(X, \rho)$ with an $\infty$-metrics $\rho: X \times X \rightarrow \overline{\mathrm{R}}_{+}$; in what follows the mapping is referred to as metrics and the space $X$ is called a metric space. We denote by $B_{X}\left(x_{0}, r\right)=\left\{x \in X: \rho\left(x, x_{0}\right) \leqslant r\right\}$ a closed ball in $X$ centered at a point $x_{0} \in X$ of a radius $r \in(0, \infty]$. We also suppose that a non-empty set $Y$ is given, on which a distance is defined, which is a mapping $d: Y \times Y \rightarrow \overline{\mathbb{R}}_{+}$ obeying the condition

$$
\forall y_{1}, y_{2} \in Y \quad d\left(y_{1}, y_{2}\right)=0 \quad \Leftrightarrow \quad y_{1}=y_{2} .
$$

In the space $Y$ we define the notion of convergence of a sequence $\left\{y_{i}\right\} \subset Y$ to an element $y \in Y$ as $i \rightarrow \infty$ by the relation

$$
y_{i} \rightarrow y \quad \Leftrightarrow \quad d\left(y_{i}, y\right) \rightarrow 0
$$

We observe that under such convergence, the limit $y$ is not necessary unique and a symmetric scalar sequence $d\left(y, y_{i}\right)$ not necessary convergence to 0 .

For the mappings acting from $X$ into $Y$ we employ the following standard definitions. A mapping $f: X \rightarrow Y$ is called continuous at a point $x \in X$ if for each sequence $\left\{x_{i}\right\} \subset X$ converging to $x$ we have $f\left(x_{i}\right) \rightarrow f(x)$. A mapping $f: X \rightarrow Y$ is called closed at a point $x \in X$ if the convergence of a sequence $\left\{x_{i}\right\} \subset X$ to $x$ and the existence of $y \in Y$ such that $f\left(x_{i}\right) \rightarrow y$ imply the identity $f(x)=y$. A mapping continuous (closed) at all points is called continuous (closed). We stress that in contrast to metric spaces, the continuity of a mapping does not imply its closedness.

We formally extend the following definitions known for usual metric spaces to the mappings in the considered spaces, see [11.

Definition 2.1. Let $\alpha>0$. A mapping $f: X \rightarrow Y$ is called $\alpha$-covering if the relation

$$
\forall x \in X \forall y \in Y \exists u \in X \quad f(u)=y, \rho(x, u) \leqslant \frac{1}{\alpha} d(f(x), y) .
$$

holds true.

Definition 2.2. Let $\beta \geqslant 0$. A mapping $f: X \rightarrow Y$ is called $\beta$-Lipschitz on a set $U \subset X$ if the relation

$$
\forall x, u \in U \quad d(f(x), f(u)) \leqslant \beta \rho(x, u)
$$

holds true. If $U=X$, then the mapping $f: X \rightarrow Y$ is called $\beta$-Lipschitz.

We are going to define weakened properties of closedness, covering and Lipschitz property for a mapping $f: X \rightarrow Y$. Given a set $U \subset X$, we define sets:

$$
\begin{gathered}
\mathrm{Cl}[f ; U]:=\left\{(x, y) \in X \times Y \mid \forall\left\{x_{n}\right\} \subset U x_{n} \rightarrow x, f\left(x_{n}\right) \rightarrow y \Rightarrow f(x)=y\right\} ; \\
\operatorname{Cov}_{\alpha}[f ; U]:=\left\{(x, y) \in X \times Y \mid \exists u \in U f(u)=y, \rho(x, u) \leqslant \alpha^{-1} d(f(x), y), \rho(x, u)<\infty\right\} ; \\
\operatorname{Lip}_{\beta}[f ; U]:=\{(x, y) \in X \times Y \mid \forall u \in U f(u)=y \Rightarrow d(f(x), y) \leqslant \beta \rho(x, u)\} .
\end{gathered}
$$


In the case $U=X$, the metrics $\rho$ and the distance $d$ have values in $\mathbb{R}_{+}$, such sets of closedness, covering and Lipschitz properties were introduced in [10].

It is obvious that relation $\mathrm{Cl}[f ; X]=X \times Y$ is equivalent to the fact that the mapping $f$ is closed, the relation $\operatorname{Cov}_{\alpha}[f ; X]=X \times Y$ means that the mapping $f$ is $\alpha$-covering, while relation $\operatorname{Lip}_{\beta}[f ; X]=X \times Y$ is valid if and only if $f$ is Lipschitz with the coefficient $\beta$.

Given a mapping $F: X \times X \rightarrow Y$ and an element $\widehat{y} \in Y$, we define a mapping $G: X \rightarrow Y$ by the identity $G(x)=F(x, x)$ and consider the equation

$$
G(x)=\widehat{y}
$$

with an unknown $x \in X$. We formulate a statement on solvability of equation (2.1) similar to Theorem 2 in [10]; this statement will be employed later.

Theorem 2.1. Let a metric space $X$ be complete and suppose that we are given $\alpha>\beta \geqslant 0$, $x_{0} \in X$ such that $d\left(F\left(x_{0}, x_{0}\right), \widehat{y}\right)<\infty$. We define:

$$
R:=(\alpha-\beta)^{-1} d\left(F\left(x_{0}, x_{0}\right), \widehat{y}\right), \quad U:=B_{X}\left(x_{0}, R\right)
$$

and assume that for each $x \in U$ the embeddings hold:

$$
(x, \widehat{y}) \in \operatorname{Cov}_{\alpha}[F(\cdot, x) ; X], \quad(x, \widehat{y}) \in \operatorname{Lip}_{\beta}[F(x, \cdot) ; U], \quad(x, \widehat{y}) \in \mathrm{Cl}[G ; U] .
$$

Then there exists a solution to equation (2.1) in the ball $U$.

We note that in a similar Theorem 2 in [10], the metrics $\rho$ and the distance $d$ were assumed to have values in $\mathbb{R}_{+}$and there employed more restrictive definitions of closedness, covering and Lipschitz property sets, namely, the corresponded to the case $U=X$. Nevertheless, for the considered here mappings $\rho$ and $d$ acting in $\overline{\mathbb{R}}_{+}$and for our definitions of the corresponding set the proof is the same as in [10] and this is why we do not provide it.

\section{FunCtional EQUATiOns in the SPACE OF MEASURABle FunCtions}

Let $\tau>0$. We denote by $\mu$ the Lebesgue measure on $[0, \tau]$, while $\mathbb{S}=\mathbb{S}([0, \tau], \mathbb{R})$ denotes the space of Lebesgue measurable functions $u:[0, \tau] \rightarrow \mathbb{R}$. In the space $\mathbb{S}$, we select a subset $\mathbb{S}_{+}$of non-negative functions. The distance in the space $\mathbb{S}$ is defined as follows.

Suppose that we are given a function $\theta: \mathbb{R} \times \mathbb{R} \rightarrow \mathbb{R}_{+}$. We assume that the following condition holds true.

$(\mathcal{A})$ The function $\theta$ is continuous with respect to each of its variables, $\theta(z, z)=0$ for each $z \in \mathbb{R}$ and the relation holds:

$$
\forall \delta>0 \exists \gamma>0 \forall z_{1}, z_{2} \in \mathbb{R} \quad\left|z_{1}-z_{2}\right| \geqslant \delta \Rightarrow \theta\left(z_{1}, z_{2}\right) \geqslant \gamma .
$$

We define a mapping $d^{\theta}: \mathbb{S} \times \mathbb{S} \rightarrow \overline{\mathbb{R}}_{+}$by the relation

$$
d^{\theta}\left(z_{1}, z_{2}\right)=\underset{t \in[0, \tau]}{\text { vrai } \sup } \theta\left(z_{1}(t), z_{2}(t)\right), \quad z_{1}, z_{2} \in \mathbb{S} .
$$

Here the composition $\theta\left(z_{1}(\cdot), z_{2}(\cdot)\right)$ is a measurable function since the function $\theta$ is continuous with respect to each of its variables. The mapping $d^{\theta}$ obviously satisfies the identity axiom, that is, this mapping is a distance in $\mathbb{S}$. The space $\left(\mathbb{S}, d^{\theta}\right)$ is denoted by $\mathbb{S}^{\theta}$. We note that the distance $d^{\theta}$ is not necessarily symmetric and does not necessary satisfy the triangle inequality.

We also observe that the function $\theta_{0}: \mathbb{R} \times \mathbb{R} \rightarrow \mathbb{R}_{+}$defined by formula

$$
\theta_{0}\left(z_{1}, z_{2}\right)=\left|z_{1}-z_{2}\right|,
$$

the corresponding mapping $d^{\theta_{0}}: \mathbb{S} \times \mathbb{S} \rightarrow \overline{\mathbb{R}}_{+}$is a metrics in $\mathbb{S}$. We denote this metrics by $\rho$, that is, $\rho=d^{\theta_{0}}$, and the corresponding space of measurable functions is denoted by $\mathbb{S}^{\theta_{0}}=(\mathbb{S}, \rho)$. The space $\mathbb{S}^{\theta_{0}}$ is complete. In this space a ball $B_{\mathbb{S}^{\theta_{0}}}\left(x_{0}, r\right)$ centered at $x_{0} \in \mathbb{S}^{\theta_{0}}$ of a radius $r \in(0, \infty]$ is a set of all measurable functions $x:[0, \tau] \rightarrow \mathbb{R}$ such that $x(t) \in B_{\mathbb{R}}\left(x_{0}(t), r\right)=$ $\left[x_{0}(t)-r, x_{0}(t)+r\right]$ for a.e. $t \in[0, \tau]$. 
Suppose that we are given a function $g:[0, \tau] \times \mathbb{R} \rightarrow \mathbb{R}$ satisfying the Caratheodory condition, that is, it is measurable with respect to the first variable and is continuous with respect to the second variable. We define a Nemytskii operator:

$$
\left(N_{g} u\right)(t)=g(t, u(t)) .
$$

By the made assumptions on the function $g$, this operator maps measurable functions into measurable ones. Let us study the closedness, continuity, covering and Lipschitz property of the operator $N_{g}$ as acting from $\mathbb{S}^{\theta_{0}}=(\mathbb{S}, \rho)$ into $\mathbb{S}^{\theta}=\left(\mathbb{S}, d^{\theta}\right)$, where the function $\theta: \mathbb{R} \times \mathbb{R} \rightarrow \mathbb{R}_{+}$ satisfies condition $(\mathcal{A})$.

Proposition 1. The operator $N_{g}: \mathbb{S}^{\theta_{0}} \rightarrow \mathbb{S}^{\theta}$ is closed. If, in addition, the set of functions $\{g(t, \cdot): \mathbb{R} \rightarrow \mathbb{R}, t \in[0, \tau]\}$ is equicontinuous, that is,

$$
\forall \varepsilon>0 \exists \delta>0 \forall t \in[0, \tau] \forall x, u \in \mathbb{R} \quad|x-u|<\delta \quad \Rightarrow \quad|g(t, x)-g(t, u)|<\varepsilon,
$$

and the set of the functions $\left\{\theta(\cdot, z): \mathbb{R} \rightarrow \mathbb{R}_{+}, z \in \mathbb{R}\right\}$ satisfies the relation

$$
\forall \varepsilon>0 \exists \delta>0 \forall z_{1}, z_{2} \in \mathbb{R} \quad\left|z_{1}-z_{2}\right|<\delta \quad \Rightarrow \quad \theta\left(z_{1}, z_{2}\right)<\varepsilon,
$$

then the operator $N_{g}: \mathbb{S}^{\theta_{0}} \rightarrow \mathbb{S}^{\theta}$ is continuous.

Proof. We are going to show that for each $z \in \mathbb{R}$ and for each sequence $\left\{z_{i}\right\} \subset \mathbb{R}$ the relation $\theta\left(z_{i}, z\right) \rightarrow 0$ is equivalent to $\left|z_{i}-z\right| \rightarrow 0$.

First, let $\theta\left(z_{i}, z\right) \rightarrow 0$. If relation $\left|z_{i}-z\right| \rightarrow 0$ does not hold, then there exists a subsequence $\left\{z_{i_{j}}\right\}$ and positive numbers $\delta$ such that $\left|z_{i_{j}}-z\right| \geqslant \delta$. By (3.1) we obtain that $\theta\left(z_{i_{j}}, z\right) \geqslant \gamma$ for some positive $\gamma$. This inequality contradicts the convergence $\theta\left(z_{i}, z\right) \rightarrow 0$. Thus, $\left|z_{i}-z\right| \rightarrow 0$.

And vice versa, by the continuity of the function $\theta(\cdot, z)$ we obtain that in the case $\left|z_{i}-z\right| \rightarrow 0$ we have $\theta\left(z_{i}, z\right) \rightarrow \theta(z, z)=0$.

Now we are going to prove the closedness of the operator $N_{g}: \mathbb{S}^{\theta_{0}} \rightarrow \mathbb{S}^{\theta}$. Suppose that we are given elements $u \in \mathbb{S}^{\theta_{0}}, y \in \mathbb{S}^{\theta}$ and a sequence $\left\{u_{i}\right\} \subset \mathbb{S}^{\theta_{0}}$ such that

$$
\begin{aligned}
& \underset{t \in[0, \tau]}{\operatorname{vrai} \sup _{0}} \theta_{0}\left(u_{i}(t), u(t)\right)=\underset{t \in[0, \tau]}{\text { vrai } \sup _{i}}\left|u_{i}(t)-u(t)\right| \rightarrow 0, \\
& \text { vrai sup } \theta\left(g\left(t, u_{i}(t)\right), y(t)\right) \rightarrow 0 .
\end{aligned}
$$

According the said above, relations (3.6) and (3.7) imply the convergences

$$
u_{i}(t) \rightarrow u(t), \quad g\left(t, u_{i}(t)\right) \rightarrow y(t) \quad \text { for a.e. } t \in[0, \tau] .
$$

Since the function $g(t, \cdot)$ is continuous, we have $g\left(t, u_{i}(t)\right) \rightarrow g(t, u(t))$ for a.e. $t \in[0, \tau]$. Then by the uniqueness of the limit in $\mathbb{R}$ we obtain $g(t, u(t))=y(t)$.

Now we assume that conditions (3.4), (3.5) hold and we are going to prove the continuity of the operator $N_{g}: \mathbb{S}^{\theta_{0}} \rightarrow \mathbb{S}^{\theta}$.

Suppose that we are given a converging sequence $\left\{u_{i}\right\} \subset \mathbb{S}^{\theta_{0}}$, that is, assertion (3.6) holds true. It follows from (3.4) and (3.6) that vrai $\sup _{t \in[0, \tau]}\left|g\left(t, u_{i}(t)\right)-g(t, u(t))\right| \rightarrow 0$, and by (3.5) we obtain relation (3.7), where $y(t)=g(t, u(t))$. Thus, we have proved that the Nemytskii operator $N_{g}: \mathbb{S}^{\theta_{0}} \rightarrow \mathbb{S}^{\theta}$ is continuous. The proof is complete.

Suppose that we are given a multi-valued mapping $\Omega:[0, \tau] \rightrightarrows \mathbb{R}$, i.e., which maps each $t \in[0, \tau]$ into a non-empty closed set $\Omega(t) \subset \mathbb{R}$. We assume that this mapping is measurable; the results on measurable multi-valued mappings can be found, for instance, in [12, Sect. 1.5]. The set of its measurable sections is denoted by

$$
\operatorname{Sel}(\Omega):=\{u \in \mathbb{S} \mid u(t) \in \Omega(t) \text { for a.e. } t \in[0, \tau]\} .
$$


Proposition 2. Suppose that we are given $x, y \in \mathbb{S}, \alpha>0$ and a measurable multi-valued mapping $\Omega:[0, \tau] \rightrightarrows \mathbb{R}$ and let for a.e. $t \in[0, \tau]$ the following condition holds:

$$
\exists u \in \Omega(t) \quad g(t, u)=y(t) \quad \text { and } \quad|x(t)-u| \leqslant \alpha^{-1} \theta(g(t, x(t)), y(t)) .
$$

Then $(x, y) \in \operatorname{Cov}_{\alpha}\left[N_{g} ; \operatorname{Sel}(\Omega)\right]$, where the operator $N_{g}: \mathbb{S}^{\theta_{0}} \rightarrow \mathbb{S}^{\theta}$ is defined by relation 3.3.

Proof. We let $r(t)=\alpha^{-1} \theta(g(t, x(t)), y(t))$. Since the function $g$ satisfies the Caratheodory conditions and the function $\theta$ is continuous in each argument, the function $[0, \tau] \ni t \mapsto r(t) \in$ $\mathbb{R}_{+}$is measurable. Now we define a multi-valued mapping

$$
[0, \tau] \ni t \mapsto \mathfrak{B}(t)=[x(t)-r(t), x(t)+r(t)],
$$

which is obviously measurable. Assertion (3.8) implies that $y(t) \in g(t, \mathfrak{B}(t) \cap \Omega(t))$ for a.e. $t \in[0, \tau]$. According Fillipov lemma, see, for instance, [12, Lm. 1.5.15], there exists a function $\widehat{u} \in S^{\theta_{0}}$ such that $\widehat{u}(t) \in \mathfrak{B}(t) \cap \Omega(t)$ and $g(t, \widehat{u}(t))=y(t)$ for a.e. $t \in[0, \tau]$. For this function we have $u \in \operatorname{Sel}(\Omega)$ and

$$
\rho(x, \widehat{u})=\operatorname{vrai}_{t \in[0, \tau]}|x(t)-\widehat{u}(t)| \leqslant \alpha^{-1} \operatorname{vrai~sup}_{t \in[0, \tau]} \theta(g(t, x(t)), y(t))=\alpha^{-1} d^{\theta}\left(N_{g} x, y\right),
$$

therefore, $(x, y) \in \operatorname{Cov}_{\alpha}\left[N_{g} ; \operatorname{Sel}(\Omega)\right]$.

Remark 1. On the real line $\mathbb{R}$ we define the distance $\theta$ and denote $\mathbb{R}^{\theta}=(\mathbb{R}, \theta)$, while the symbol $\mathbb{R}$ stands for the real space with the usual metrics $\theta_{0}$. The function $g$ can be regarded as a mapping $[0, \tau] \times \mathbb{R} \rightarrow \mathbb{R}^{\theta}$. Then relation (3.8) means that

$$
(x(t), y(t)) \in \operatorname{Cov}_{\alpha}[g(t, \cdot) ; \Omega(t)], \quad g(t, \cdot): \mathbb{R} \rightarrow \mathbb{R}^{\theta}, \quad t \in[0, \tau] .
$$

Thus, Proposition 2 can be formulated as follows: if for some $x, y \in \mathbb{S}$ and $\alpha>0$ belonging (3.9) holds, then $(x, y) \in \operatorname{Cov}_{\alpha}\left[N_{g} ; \operatorname{Sel}(\Omega)\right], N_{g}: \mathbb{S}^{\theta_{0}} \rightarrow \mathbb{S}^{\theta}$.

Example 1. We define a function $\theta: \mathbb{R} \times \mathbb{R} \rightarrow \mathbb{R}_{+}$by the relations

$$
\begin{aligned}
& z_{1} z_{2} \geqslant 0 \Rightarrow \theta\left(z_{1}, z_{2}\right)= \begin{cases}\mid \sqrt{\left|z_{1}\right|}-\sqrt{\left|z_{2}\right|} & \text { if } \sqrt{\left|z_{1}\right|}+\sqrt{\left|z_{2}\right|} \leqslant 1, \\
\left|z_{1}-z_{2}\right| & \text { if } \sqrt{\left|z_{1}\right|}+\sqrt{\left|z_{2}\right|}>1\end{cases} \\
& z_{1} z_{2}<0 \Rightarrow \theta\left(z_{1}, z_{2}\right)=\theta\left(z_{1}, 0\right)+\theta\left(0, z_{2}\right) .
\end{aligned}
$$

This function satisfies condition $(\mathcal{A})$.

We mention one more property of the function $\theta$ obviously implied by its definition:

$$
\forall \lambda \in[0,1) \quad \forall z_{1}, z_{2} \in \mathbb{R} \quad \lambda \theta\left(z_{1}, z_{2}\right) \leqslant \theta\left(\lambda z_{1}, \lambda z_{2}\right) \leqslant \sqrt{\lambda} \theta\left(z_{1}, z_{2}\right) .
$$

Indeed, in the case $z_{1} z_{2} \geqslant 0$ we have

$$
\begin{aligned}
& \sqrt{\left|z_{1}\right|}+\sqrt{\left|z_{2}\right|} \leqslant 1 \Rightarrow \theta\left(\lambda z_{1}, \lambda z_{2}\right)=\sqrt{\lambda} \mid \sqrt{\left|z_{1}\right|}-\sqrt{\left|z_{2}\right|}=\sqrt{\lambda} \theta\left(z_{1}, z_{2}\right) \Rightarrow(3.12) ; \\
& 1<\sqrt{\left|z_{1}\right|}+\sqrt{\left|z_{2}\right|} \leqslant 1 / \sqrt{\lambda} \Rightarrow \theta\left(\lambda z_{1}, \lambda z_{2}\right)=\sqrt{\lambda} \frac{\left|z_{1}-z_{2}\right|}{\sqrt{\left|z_{1}\right|}+\sqrt{\left|z_{2}\right|} \Rightarrow 3.12} ; \\
& \sqrt{\left|z_{1}\right|}+\sqrt{\left|z_{2}\right|}>1 / \sqrt{\lambda} \Rightarrow \theta\left(\lambda z_{1}, \lambda z_{2}\right)=\lambda\left|z_{1}-z_{2}\right|=\lambda \theta\left(z_{1}, z_{2}\right) \Rightarrow 3.12 .
\end{aligned}
$$

And if $z_{1} z_{2}<0$, then $\theta\left(\lambda z_{1}, \lambda z_{2}\right)=\theta\left(\lambda z_{1}, 0\right)+\theta\left(0, \lambda z_{2}\right)$, and therefore,

$$
\begin{aligned}
& \theta\left(\lambda z_{1}, \lambda z_{2}\right) \leqslant \sqrt{\lambda} \theta\left(z_{1}, 0\right)+\sqrt{\lambda} \theta\left(0, z_{2}\right)=\sqrt{\lambda} \theta\left(z_{1}, z_{2}\right) ; \\
& \theta\left(\lambda z_{1}, \lambda z_{2}\right) \geqslant \lambda \theta\left(z_{1}, 0\right)+\lambda \theta\left(0, z_{2}\right)=\lambda \theta\left(z_{1}, z_{2}\right) .
\end{aligned}
$$

Above relation 3.12 is equivalent to

$$
\forall \nu>1 \quad \forall z_{1}, z_{2} \in \mathbb{R} \quad \sqrt{\nu} \theta\left(z_{1}, z_{2}\right) \leqslant \theta\left(\nu z_{1}, \nu z_{2}\right) \leqslant \nu \theta\left(z_{1}, z_{2}\right) .
$$


Let $\tau=1, \mathbb{S}=\mathbb{S}([0,1], \mathbb{R})$. In terms of the function $\theta$ given by relations $(3.10),(3.11)$, we introduce the distance $d^{\theta}$ in the space $\mathbb{S}$ by formula $(3.2)$. This distance is symmetric and satisfies triangle inequality, that is, it is an $\infty$-metrics.

First of all we note that by the inequality $\theta\left(z_{1}, z_{2}\right) \geqslant\left|z_{1}-z_{2}\right|, z_{1}, z_{2} \in \mathbb{R}$, we have $d^{\theta}\left(x_{1}, x_{2}\right) \geqslant$ $d^{\theta_{0}}\left(x_{1}, x_{2}\right), x_{1}, x_{2} \in \mathbb{S}$. Therefore, each mapping in the space $\mathbb{S}$ being $\alpha$-covering with respect to the usual metrics $d^{\theta_{0}}$, i.e., as a mapping $\mathbb{S}^{\theta_{0}} \rightarrow \mathbb{S}^{\theta_{0}}$, is also a $\alpha$-covering as a mapping $\mathbb{S}^{\theta_{0}} \rightarrow \mathbb{S}^{\theta}$. We consider a mapping which is not covering with any constant $\alpha$ if it is regarded as a mapping $\mathbb{S}^{\theta_{0}} \rightarrow \mathbb{S}^{\theta_{0}}$ and nevertheless, it is 1-covering as a mapping $\mathbb{S}^{\theta_{0}} \rightarrow \mathbb{S}^{\theta}$.

Suppose that we are given a function $q \in \mathbb{S}$ such that $q(t) \geqslant 1$ for a.e. $t \in[0,1]$. We consider functions $g_{0}, g_{1},:[0,1] \times \mathbb{R} \rightarrow \mathbb{R}$ defined for each $t \in[0,1], x \in \mathbb{R}$, by the formulae

$$
g_{0}(t, x)=x^{2}, \quad g_{1}(t, x)=q(t) x^{2} .
$$

According Proposition 1, the Nemytskii operators $N_{g_{0}}, N_{g_{1}}: \mathbb{S}^{\theta_{0}} \rightarrow \mathbb{S}^{\theta}$ are closed. We first study the covering set $\operatorname{Cov}_{\alpha}\left[N_{g_{0}} ; \mathbb{S}\right]$ of the Nemytskii operator $N_{g_{0}}$ generated by the function $g_{0}$. We note that the function of the first variable $g_{0}(\cdot, x)$ is constant, the function of the second variable $g_{0}(t, \cdot)$ is even, its restriction on $\mathbb{R}_{+}$is injective and monotone and as acting in $\mathbb{R}_{+}$, it is also surjective. Let us show that for $\alpha=1$, all functions $x \in \mathbb{S}$ and $y \in \mathbb{S}_{+}$, the assumptions of Proposition 2 are satisfied, where $\Omega(t) \equiv \mathbb{R}$, that is, we are going to show the validity of relation (3.8).

Let $t \in[0,1]$. For simplicity we suppose that $x(t) \geqslant 0$. We define $u=\sqrt{y(t)}$; if $x(t)<0$, then we let $u=-\sqrt{y(t)}$. We have:

$$
\begin{aligned}
& x(t)+u \leqslant 1 \Rightarrow \theta\left(g_{0}(t, x(t)), y(t)\right)=|x(t)-u| ; \\
& x(t)+u>1 \Rightarrow \theta\left(g_{0}(t, x(t)), y(t)\right)=\left|x^{2}(t)-u^{2}\right|=|x(t)-u|(x(t)+u) \geqslant|x(t)-u| .
\end{aligned}
$$

Thus, relation $(3.8)$ is valid and hence, according Proposition 2 , we obtain:

$$
\forall x \in \mathbb{S}, \quad \forall y \in \mathbb{S}_{+} \quad(x, y) \in \operatorname{Cov}_{\alpha}\left[N_{g_{0}} ; \mathbb{S}\right], \quad \text { where } \quad \alpha=1 .
$$

Let us show that for the Nemytskii operator $N_{g_{1}}: \mathbb{S}^{\theta_{0}} \rightarrow \mathbb{S}^{\theta}$ generated by the function $g_{1}$, the set $\operatorname{Cov}_{\alpha}\left[N_{g_{1}} ; \mathbb{S}\right]$ for $\alpha=1$ also contains all pairs $(x, y) \in \mathbb{S} \times \mathbb{S}_{+}$. Since $\left(x(\cdot), q^{-1}(\cdot) y(\cdot)\right) \in$ $\operatorname{Cov}_{\alpha}\left[N_{g_{0}} ; \mathbb{S}\right]$, there exists a function $u \in \mathbb{S}$ such that

$$
\left(N_{g_{0}} u\right)(\cdot)=q^{-1}(\cdot) y(\cdot) \Leftrightarrow N_{g_{1}} u=y
$$

and the inequalities hold:

$$
\rho(x, u) \leqslant d^{\theta}\left(N_{g_{0}} x, q^{-1}(\cdot) y(\cdot)\right)=d^{\theta}\left(q^{-1}(\cdot)\left(N_{g_{1}} x\right)(\cdot), q^{-1}(\cdot) y(\cdot)\right) .
$$

On the base of relation 3.12 we obtain inequality $\rho(x, u) \leqslant d^{\theta}\left(N_{g_{1}} x, y\right)$. Thus, we have proved that $(x, y) \in \operatorname{Cov}_{\alpha}\left[N_{g_{1}} ; \mathbb{S}\right]$.

Example 2. We consider one more function $\theta: \mathbb{R} \times \mathbb{R} \rightarrow \mathbb{R}_{+}$satisfying condition $(\mathcal{A})$ and defined by relations:

$$
\begin{aligned}
& z_{1} z_{2} \geqslant 0 \Rightarrow \theta\left(z_{1}, z_{2}\right)= \begin{cases}\left|z_{1}^{2}-z_{2}^{2}\right|, & \text { if }\left|z_{1}+z_{2}\right| \leqslant 1 \\
\left|z_{1}-z_{2}\right|, & \text { if }\left|z_{1}+z_{2}\right|>1\end{cases} \\
& z_{1} z_{2}<0 \Rightarrow \theta\left(z_{1}, z_{2}\right)=\theta\left(z_{1}, 0\right)+\theta\left(0, z_{2}\right)
\end{aligned}
$$

In the space $\mathbb{S}=\mathbb{S}([0,1], \mathbb{R})$, we define the distance $d^{\theta}$ by formula 3.2 . This distance is symmetric and nevertheless, it is not metrics since it does not satisfy the triangle inequality; for instance, for $z_{1}=0, z_{2}=\frac{1}{2}, z_{3}=1$ we have

$$
\theta\left(z_{1}, z_{2}\right)=\frac{1}{4}, \quad \theta\left(z_{2}, z_{3}\right)=\frac{1}{2}, \quad \theta\left(z_{1}, z_{3}\right)=1>\frac{1}{4}+\frac{1}{2} .
$$


We consider a function $\widehat{g}_{0}:[0,1] \times \mathbb{R} \rightarrow \mathbb{R}$,

$$
\widehat{g}_{0}(t, x)=|x|+\sqrt{|x|}, \quad x \in \mathbb{R}, \quad t \in[0,1] .
$$

It is easy to confirm, by the same arguing as in Example 1, that for $\alpha=1$, each $x \in \mathbb{S}$ and $y \in \mathbb{S}_{+}$, the assumptions of Proposition 2 are satisfied, where $\Omega(t) \equiv \mathbb{R}$. Thus,

$$
\forall(x, y) \in \mathbb{S} \times \mathbb{S}_{+} \quad(x, y) \in \operatorname{Cov}_{\alpha}\left[N_{\widehat{g}_{0}} ; \mathbb{S}\right], \quad \text { where } \quad \alpha=1 .
$$

Proposition 3. Suppose that we are given $x, y \in \mathbb{S}, \beta \geqslant 0$ and a measurable multi-valued mapping $\Omega:[0, \tau] \rightrightarrows \mathbb{R}$ and for a.e. $t \in[0, \tau]$ the implication

$$
\forall u \in \Omega(t) \quad g(t, u)=y(t) \Rightarrow \theta(g(t, x(t)), y(t)) \leqslant \beta|x(t)-u|
$$

holds true. Then $(x, y) \in \operatorname{Lip}_{\beta}\left[N_{g} ; \operatorname{Sel}(\Omega)\right]$, where the operator $N_{g}: S^{\theta_{0}} \rightarrow \mathbb{S}^{\theta}$ is defined by relation 3.3 .

Proof. Let for some function $\widehat{u} \in \operatorname{Sel}(\Omega)$ the identity $N_{g} \widehat{u}=y$ holds true. Then it follows from relation (3.17) that

$$
d^{\theta}\left(N_{g} x, y\right)=\underset{t \in[0, \tau]}{\operatorname{vrai} \sup _{0}} \theta(g(t, x(t)), y(t)) \leqslant \beta \text { vrai } \sup _{t \in[0, \tau]}|x(t)-\widehat{u}(t)|=\beta \rho(x, \widehat{u}) .
$$

Thus, $(x, y) \in \operatorname{Lip}_{\beta}\left[N_{g} ; \operatorname{Sel}(\Omega)\right]$. The proof is complete.

Remark 2. Implication (3.17) means that the belonging

$$
(x(t), y(t)) \in \operatorname{Lip}_{\beta}[g(t, \cdot), \Omega(t)], \quad g(t, \cdot): \mathbb{R} \rightarrow \mathbb{R}^{\theta}, \quad t \in[0, \tau],
$$

holds. This is why Proposition 2 can be formulated as follows: if for some $x, y \in \mathbb{S}$ and $\beta \geqslant 0$ belonging (3.18) holds, then $(x, y) \in \operatorname{Lip}_{\beta}\left[N_{g} ; \operatorname{Sel}(\Omega)\right], N_{g}: \mathbb{S}^{\theta_{0}} \rightarrow \mathbb{S}^{\theta}$.

Corollary 1. Suppose that we are given a multi-valued mapping $\Omega:[0, \tau] \rightrightarrows \mathbb{R}$. Assume that for a.e. $t \in[0, \tau]$ the mapping $g(t, \cdot): \mathbb{R} \rightarrow \mathbb{R}^{\theta}$ is $\beta$-Lipschitz on the set $\Omega(t)$, that is, for all $x, u \in \Omega(t)$ the inequality holds:

$$
\theta(g(t, x), g(t, u)) \leqslant \beta|x-u| .
$$

Then for all $x \in \operatorname{Sel}(\Omega), y \in \mathbb{S}^{\theta}$ we have $(x, y) \in \operatorname{Lip}_{\beta}\left[N_{g} ; \operatorname{Sel}(\Omega)\right]$, where $N_{g}: \mathbb{S}^{\theta_{0}} \rightarrow \mathbb{S}^{\theta}$, that is, the operator $N_{g}$ is $\beta$-Lipschitz on the set $\operatorname{Sel}(\Omega)$.

Example 3. As in Example 1, we define a function $\theta$ by formulae (3.10), (3.11). By the inequality $\theta\left(z_{1}, z_{2}\right) \geqslant\left|z_{1}-z_{2}\right|$, each function $g:[0,1] \times \mathbb{R} \rightarrow \mathbb{R}$ obeying condition (3.19) for some $\beta \geqslant 0$ satisfies also usual Lipschitz condition:

$$
|g(t, x)-g(t, u)| \leqslant \beta|x-u|, \quad x, u \in \Omega(t) .
$$

The opposite statement is false. Since for the function $g(t, x)=x$ relation 3.20 holds as $\Omega(t) \equiv \mathbb{R}$ with the Lipschitz coefficient being equal to 1 but equation (3.19) fails for each $\beta \geqslant 0$ even if $\Omega(t) \equiv[0, \varepsilon]$, where $\varepsilon>0$ is arbitrary small. Indeed, for each $x>0$ we have

$$
\theta(g(t, x), g(t, 0))=\sqrt{x}=\beta_{x}|x-0|,
$$

where $\beta_{x}=1 / \sqrt{x} \rightarrow \infty$ as $x \rightarrow 0+$.

Now we consider a function obeying condition (3.19). Suppose that we are given a function $p \in \mathbb{S}$ such that $p(t) \geqslant 1 / 2$ for a.e. $t \in[0,1]$ and a number $\beta \geqslant 0$. We let

$$
g_{2}:[0,1] \times \mathbb{R} \rightarrow \mathbb{R}, \quad g_{2}(t, x)=\beta|x|+p(t), \quad x \in \mathbb{R}, \quad t \in[0,1] .
$$

The values of this function satisfies the inequality $g_{2}(t, x) \geqslant 1 / 2$ for all $t, x$ and this is why by formula 3.10 we have:

$$
\theta\left(g_{2}(t, x), g_{2}(t, u)\right)=\left|g_{2}(t, x)-g_{2}(t, u)\right|=\beta|| x|-| u|| \leqslant \beta|x-u|, \quad x, u \in \mathbb{R}, \quad t \in[0,1] .
$$


Hence, the assumptions of Corollary 1 are satisfied with $\Omega(t) \equiv \mathbb{R}$ and this is why the Nemytskii operator $N_{g_{2}}: \mathbb{S}^{\theta_{0}} \rightarrow \mathbb{S}^{\theta}$ defined by the function $g_{2}$ is Lipschitz with a constant $\beta$ on entire space $\mathbb{S}$. We also note that according Proposition 1 , the operator $N_{g_{2}}$ is closed.

Example 4. As in Example 2, we define a function $\theta$ by formulae (3.14), (3.15) and a function $\widehat{g}_{0}:[0,1] \times \mathbb{R} \rightarrow \mathbb{R}$ by relation (3.16). This function satisfies inequality $(3.19)$ with the coefficient $\beta=4$ for all $x, u \in \mathbb{R}$. According Corollary 1 , the Nemytskii operator $N_{\widehat{g}_{0}}: \mathbb{S}^{\theta_{0}} \rightarrow \mathbb{S}^{\theta}$ defined by the function $\widehat{g}_{0}$ is Lipschitz with constant $\beta=4$ on entire space $\mathbb{S}$.

Let us formulate conditions ensuring Lipschitz property for one more mapping, which will be used in studying various functional equations with deviating variable.

Suppose we are given a function $h:[0, \tau] \rightarrow[0, \tau]$ such that

$$
\forall E \subset[0, \tau] \mu(E)=0 \Rightarrow \mu\left(h^{-1}(E)\right)=0 .
$$

This condition ensures the measurability of the function $u(h(\cdot))$ for each measurable function $u:[0, \tau] \rightarrow \mathbb{R}$, see [13], [14, Sect. 1.3] and this allows us to define the operator

$$
S_{h}: \mathbb{S} \rightarrow \mathbb{S}, \quad\left(S_{h} u\right)(t)=u(h(t)), \quad t \in[0, \tau] .
$$

Employing Proposition 3 , let us study the set $\operatorname{Lip}_{\beta}\left[N_{g} S_{h}\right]$ for the composition

$$
N_{g} S_{h}: \mathbb{S}^{\theta_{0}} \rightarrow \mathbb{S}^{\theta}, \quad\left(N_{g} S_{h} x\right)(t)=g(t, x(h(t))), \quad t \in[0, \tau] .
$$

In order to do this, we shall need the following statement.

Lemma 3.1. For a measurable multi-valued mapping $\Omega:[0, \tau] \rightrightarrows \mathbb{R}$, the composition $\Omega h$ : $[0, \tau] \rightrightarrows \mathbb{R}$ is also measurable. If the function $\omega \in \mathbb{S}$ is a section of the mapping $\Omega$, that is, $\omega(t) \in \Omega(t)$ for a.e. $t \in[0, \tau]$, then the function $S_{h} \omega$ is measurable and is a section of the mapping $\Omega h$, i.e., $\omega(h(t)) \in \Omega(h(t))$ for a.e. $t \in[0, \tau]$.

Proof. A multi-valued mapping is measurable if and only if it possesses Castaing representation, see [12, Thm. 1.5.6, Rem. 1.5.7], and this is why there exists a countable set of measurable sections $\omega_{n}, n=1,2, \ldots$, of the mapping $\Omega$ such that

$$
\Omega(t)=\overline{\bigcup_{n=1}^{\infty}\left\{\omega_{n}(t)\right\} \quad \text { for a.e. } \quad t \in[0, \tau] ;}
$$

here the bar denotes the closure of a set in the space $\mathbb{R}$. By condition (3.22), the functions $S_{h} \omega_{n}, n=1,2, \ldots$, are measurable. Let us prove the relation

$$
\Omega(h(t))=\overline{\bigcup_{n=1}^{\infty}\left\{\omega_{n}(h(t))\right\}} \quad \text { for a.e. } t \in[0, \tau] .
$$

We define a set

$$
I=\left\{t \in[0, \tau] \mid \Omega(h(t)) \neq \overline{\bigcup_{n=1}^{\infty}\left\{\omega_{n}(h(t))\right\}}\right\} .
$$

We obviously have $I=h^{-1}(E)$, where

$$
E=\left\{s \in[0, \tau] \mid \Omega(s) \neq \bigcup_{n=1}^{\infty}\left\{\omega_{n}(s)\right\}\right\} .
$$

Since $\mu(E)=0$, by condition 3.22 we have $\mu(I)=0$. Thus, relation $(3.24)$ is satisfied and the multi-valued mapping $\Omega h:[0, \tau] \rightrightarrows \mathbb{R}$ possesses the Castaing representation. Hence, this mapping is measurable.

Suppose that for some function $\omega \in \mathbb{S}$, for a.e. $t \in[0, \tau]$ the belonging holds $\omega(t) \in \Omega(t)$. We define a set

$$
I=\{t \in[0, \tau] \mid \omega(h(t)) \notin \Omega(h(t))\}
$$


We represent this set as $I=h^{-1}(E)$, where

$$
E=\{s \in[0, \tau] \mid \omega(s) \notin \Omega(s)\} .
$$

Since $\mu(E)=0$, by condition 3.22 we have $\mu(I)=0$. Thus, $\omega(h(t)) \in \Omega(h(t))$ for a.e. $t \in[0, \tau]$. The proof is complete.

Proposition 4. Suppose that we are given $x, y \in \mathbb{S}, \beta \geqslant 0$ and a measurable multivalued mapping $\Omega:[0, \tau] \rightrightarrows \mathbb{R}$. Let for a.e. $t \in[0, \tau]$, the belonging $(x(h(t)), y(t)) \in$ $\operatorname{Lip}_{\beta}[g(t, \cdot), \Omega(h(t))], g(t, \cdot): \mathbb{R} \rightarrow \mathbb{R}^{\theta}$ holds, that is, the assertion

$$
\forall u \in \Omega(h(t)) \quad g(t, u)=y(t) \Rightarrow \theta(g(t, x(h(t))), y(t)) \leqslant \beta|x(h(t))-u|
$$

is valid. Then $(x, y) \in \operatorname{Lip}_{\beta}\left[N_{g} S_{h} ; \operatorname{Sel}(\Omega)\right]$, where $N_{g} S_{h}: \mathbb{S}^{\theta_{0}} \rightarrow \mathbb{S}^{\theta}$ is defined by relation (3.23).

Proof. Let some function $\widehat{u} \in \operatorname{Sel}(\Omega)$ satisfies the identity $N_{g} S_{h} \widehat{u}=y$. According Lemma 3.1. $\widehat{u}(h(t)) \in \Omega(h(t))$ for a.e. $t \in[0, \tau]$. It follows from relation 3.25 that

$$
d^{\theta}\left(N_{g} S_{h} x, y\right)=\underset{t \in[0, \tau]}{\operatorname{vrai} \sup _{0}} \theta(g(t, x(h(t))), y(t)) \leqslant \beta \text { vrai } \sup _{t \in[0, \tau]}|x(h(t))-\widehat{u}(h(t))| .
$$

We define a set

$$
I=\{t \in[0, \tau]|| x(h(t))-\widehat{u}(h(t)) \mid>\rho(x, \widehat{u})\} .
$$

We represent this set as $I=h^{-1}(E), E=\{s \in[0, \tau]|| x(s)-\widehat{u}(s) \mid>\rho(x, \widehat{u})\}$. Since $\mu(E)=0$, we obtain $\mu(I)=0$ and this is why $|x(h(t))-\widehat{u}(h(t))| \leqslant \rho(x, \widehat{u})$ for a.e. $t \in[0, \tau]$. Taking into consideration this inequality, by relation 3.26 we get:

$$
d^{\theta}\left(N_{g} S_{h} x, y\right) \leqslant \beta \rho(x, \widehat{u}) .
$$

Thus, $(x, y) \in \operatorname{Lip}_{\beta}\left[N_{g} S_{h} ; \operatorname{Sel}(\Omega)\right]$. The proof is complete.

Corollary 2. Suppose we are given a multi-valued mapping $\Omega:[0, \tau] \rightrightarrows \mathbb{R}$. Assume that for a.e. $t \in[0, \tau]$, the mapping $g(t, \cdot): \mathbb{R} \rightarrow \mathbb{R}^{\theta}$ is $\beta$-Lipschtiz on the set $\Omega(h(t))$, that is, for all $x, u \in \Omega(h(t))$, inequality 3.19 holds true. Then the operator $N_{g} S_{h}: \mathbb{S}^{\theta_{0}} \rightarrow \mathbb{S}^{\theta}$ defined by relation (3.23) satisfies the belonging $(x, y) \in \operatorname{Lip}_{\beta}\left[N_{g} S_{h} ; \operatorname{Sel}(\Omega)\right]$ for all $x \in \operatorname{Sel}(\Omega), y \in \mathbb{S}^{\theta}$, that is, the operator $N_{g} S_{h}$ is a $\beta$-Lipschitz on the set $\operatorname{Sel}(\Omega)$.

Example 5. Let a function $\theta: \mathbb{R} \times \mathbb{R} \rightarrow \mathbb{R}_{+}$be defined by formulae (3.10), (3.11), and a function $g_{2}:[0,1] \times \mathbb{R} \rightarrow \mathbb{R}$ is defined by relation (3.21), where $\beta \geqslant 0$ and $p(t) \geqslant 1 / 2$ for a.e. $t \in[0,1]$. As it was shown in example 3 , this function satisfies condition $(3.19)$ for all $x, u \in \mathbb{R}$. This is why, by Corollary 2, the composition

$$
N_{g_{2}} S_{h}: \mathbb{S}^{\theta_{0}} \rightarrow \mathbb{S}^{\theta}, \quad\left(N_{g_{2}} S_{h} x\right)(t)=\beta|x(h(t))|+p(t),
$$

is a $\beta$-Lipschitz operator on entire space $\mathbb{S}$.

We are going to apply the obtained statement to studying a functional equation with a deviating variable. Suppose that we are given a function $f:[0, \tau] \times \mathbb{R} \times \mathbb{R} \rightarrow \mathbb{R}$ measurable in the first variable and jointly continuous in the second and third variables, a function $h$ : $[0, \tau] \rightarrow[0, \tau]$ obeying condition 3.22 and a measurable function $\widehat{y}:[0, \tau] \rightarrow \mathbb{R}$. We consider an equation

$$
f(t, x(h(t)), x(t))=\widehat{y}(t), \quad t \in[0, \tau]
$$

for an unknown measurable function $x:[0, \tau] \rightarrow \mathbb{R}$.

For an arbitrary function $v \in \mathbb{S}$, we define functions $g_{1}^{[v]}, g_{2}^{[v]}:[0, \tau] \times \mathbb{R} \rightarrow \mathbb{R}$ by relations

$$
g_{1}^{[v]}(t, x)=f(t, v(h(t)), x), \quad g_{2}^{[v]}(t, x)=f(t, x, v(t)), \quad t \in[0, \tau], \quad x \in \mathbb{R} .
$$

The functions $g_{1}^{[v]}, g_{2}^{[v]}$ obviously satisfy the Caratheodory condition. 
Theorem 3.1. Suppose that we are given $\alpha>\beta \geqslant 0, x_{0} \in \mathbb{S}$ such that

$$
R:=\frac{1}{\alpha-\beta} \operatorname{vrai} \sup _{t \in[0, \tau]} \theta\left(f\left(t, x_{0}(h(t)), x_{0}(t)\right), \widehat{y}(t)\right)<\infty .
$$

Let for each $v \in B_{\mathbb{S}^{\theta_{0}}}\left(x_{0}, R\right)$, the function $g_{1}^{[v]}$ satisfies condition (3.8) for each $x \in B_{\mathbb{S}_{0}}\left(x_{0}, R\right)$, given $y=\widehat{y}$ and $\Omega(t) \equiv \mathbb{R}$, while the function $g_{2}^{[v]}$ satisfies condition (3.25) with the same functions $x, y$, but with another multi-valued mapping: $\Omega(t)=B_{\mathbb{R}}\left(x_{0}(t), R\right)$ for a.e. $t \in[0, \tau]$. Then there exists a solution $\widehat{x} \in B_{\mathbb{S}_{0}}\left(x_{0}, R\right)$ to equation (3.27).

Proof. We denote by $N_{f}: \mathbb{S}^{\theta_{0}} \times \mathbb{S}^{\theta_{0}} \rightarrow S^{\theta}$ the Nemytskii operator

$$
\left(N_{f}(x, u)\right)(t)=f(t, u(t), x(t)), \quad t \in[0, \tau]
$$

and we define the mappings

$$
F: \mathbb{S}^{\theta_{0}} \times \mathbb{S}^{\theta_{0}} \rightarrow S^{\theta}, \quad F(x, u)=N_{f}\left(x, S_{h} u\right) ; \quad G: \mathbb{S}^{\theta_{0}} \rightarrow \mathbb{S}^{\theta}, \quad G(x)=F(x, x) .
$$

We are going to prove that the mappings $F, G$ are closed. We choose arbitrary sequences $\left\{x_{i}\right\},\left\{u_{i}\right\} \subset \mathbb{S}^{\theta_{0}}$, elements $x, u \in \mathbb{S}^{\theta_{0}}$ and $w \in \mathbb{S}^{\theta}$ satisfy $\rho\left(x_{i}, x\right) \rightarrow 0, \rho\left(u_{i}, u\right) \rightarrow 0$ and $d^{\theta}\left(F\left(x_{i}, u_{i}\right), w\right) \rightarrow 0$ as $i \rightarrow \infty$. Then, as it has been shown in the proof of Proposition 1 , for a.e. $t \in[0, \tau]$, the convergences hold: $x_{i}(t) \rightarrow x(t), u_{i}(t) \rightarrow u(t)$ and $\left(F\left(x_{i}, u_{i}\right)\right)(t) \rightarrow w(t)$. By the second relation, for a.e. $t \in[0, \tau]$ we have $u_{i}(h(t)) \rightarrow u(h(t))$. This is why, by the continuity of the function $f(t, \cdot, \cdot)$, for a.e. $t \in[0, \tau]$ we have $f\left(t, u_{i}(h(t)), x_{i}(t)\right) \rightarrow f(t, u(h(t)), x(t))$. Thus, $\left(F\left(x_{i}, u_{i}\right)\right)(t) \rightarrow(F(x, u))(t)$ and $\left(F\left(x_{i}, u_{i}\right)\right)(t) \rightarrow w(t)$, and hence, $(F(x, u))(t)=w(t)$, $t \in[0, \tau]$. We have proved that the mapping $F$ is closed and therefore, the mapping $G$ is also closed.

For an arbitrary function $v \in B_{\mathbb{S}_{0}}\left(x_{0}, R\right)$, the operator $F(\cdot, v): \mathbb{S}^{\theta_{0}} \rightarrow \mathbb{S}^{\theta}$ is the Nemytskii operator $N_{g_{1}^{[v]}}$ generated by the function $g_{1}^{[v]}$. This operator satisfies the assumptions of Proposition 2 with $y=\widehat{y}$, arbitrary $x \in B_{\mathbb{S}_{0}}\left(x_{0}, R\right)$ and a multi-valued mapping $t \in[0, \tau] \mapsto \Omega(t)=\mathbb{R}$. According Proposition 2 , for each $x \in B_{\mathbb{S}_{0}}\left(x_{0}, R\right)$, a pair $(x, \widehat{y})$ belongs to the set $\operatorname{Cov}_{\alpha}[F(\cdot, v) ; \mathbb{S}]$. Therefore, a pair $(v, \widehat{y})$ also belongs to the set $\operatorname{Cov}_{\alpha}[F(\cdot, v) ; \mathbb{S}]$.

The operator $F(v, \cdot): \mathbb{S}^{\theta_{0}} \rightarrow \mathbb{S}^{\theta}$ is a composition $N_{g_{2}^{[v]}} S_{h}$ satisfying the assumptions of Proposition 4 for $y=\widehat{y}$, each $x \in B_{\mathbb{S}_{0}}\left(x_{0}, R\right)$ and a multi-valued mapping $t \in[0, \tau] \mapsto$ $\Omega(t)=B_{\mathbb{R}}\left(x_{0}(t), R\right)$. By Proposition 4, for each $x \in B_{\mathbb{S}_{0}}\left(x_{0}, R\right)$, we have $(x, \widehat{y}) \in$ $\operatorname{Lip}_{\beta}\left[F(v, \cdot) ; B_{\mathbb{S}_{0}}\left(x_{0}, R\right)\right]$. Therefore, $(v, \widehat{y}) \in \operatorname{Lip}_{\beta}\left[F(u, \cdot) ; B_{\mathbb{S}_{0}}\left(x_{0}, R\right)\right]$.

In conclusion we recall that the space $\mathbb{S}^{\theta_{0}}$ is complete. Thus, all assumptions of Theorem 2.1 are satisfied and according this theorem, there exists a solution $\widehat{x} \in B_{\mathbb{S}_{0}}\left(x_{0}, R\right)$ to equation 3.27 .

Remark 3. It is assumed in Theorem 3.1 that the function $g_{2}^{[v]}$ satisfies condition (3.25), where $y=\widehat{y}, x \in B_{\mathbb{S}_{0} \theta_{0}}\left(x_{0}, R\right)$ and $\Omega(t)=B_{\mathbb{R}}\left(x_{0}(t), R\right)$. According Corollary 2, to ensure this condition, it is sufficient the mapping $g_{2}^{[v]}(t, \cdot): \mathbb{R} \rightarrow \mathbb{R}^{\theta}$ to be $\beta$-Lipschitz on the set $\left[x_{0}(h(t))-R, x_{0}(h(t))+R\right]$ for a.e. $t \in[0, \tau]$.

Example 6. Suppose that we are given functions $p, \widehat{y} \in \mathbb{S}_{+}, \gamma \geqslant 0$ and a function $h:[0,1] \rightarrow$ $[0,1]$ obeying condition 3.22 with $\tau=1$. We consider the equation

$$
x^{2}(t)(p(t)+\gamma x(h(t)))=\widehat{y}(t), \quad t \in[0,1] .
$$

We are interesting in existence of a non-negative solution to this equation belonging to some neighbourhood of the function $x_{0}(t) \equiv 0$ in the space $\mathbb{S}$. The mappings

$$
x(\cdot) \in \mathbb{S} \mapsto x^{2}(\cdot) \in \mathbb{S}, \quad x(\cdot) \in \mathbb{S} \mapsto x(h(t)) \in \mathbb{S},
$$


involved in the left hand side of equation $(3.29)$ are not $\alpha$-coverging with respect to the usual metrics $d^{\theta_{0}}$ in the space $\mathbb{S}$ for all $\alpha>0$. Thus, we can not apply theorems on such mappings to this equation. Let us demonstrate the abilities of Theorem 3.1 in studying equation (3.29).

We let

$$
R=2 \operatorname{vrai} \sup _{t \in[0,1]} \widehat{y}(t) .
$$

We are going to show that under the conditions

$$
p(t) \geqslant 1 \text { for a.e. } t \in[0,1] ; \quad 2 \gamma R<1 \quad \text { and } \quad 2 \gamma R^{2}<1,
$$

equation $(3.29)$ possesses a solution $x \in \mathbb{S}_{+}$such that $x(t) \leqslant R$ for a.e. on $[0,1]$.

We define an auxiliary equation

$$
x^{2}(t)(p(t)+\gamma|x(h(t))|)=\widehat{y}(t), \quad t \in[0,1] .
$$

For each solution $x \in \mathbb{S}$ of equation (3.31), the function $|x(\cdot)|$ is a solution of equation (3.29), and by a solution $x \in \mathbb{S}_{+}$of equation $(3.29)$ we obviously find a solution to equation (3.31). Thus, the solvability of equations (3.29), (3.31) in $\mathbb{S}_{+}$are equivalent, but the domain of the function

$$
f\left(t, x_{1}, x_{2}\right)=x_{2}^{2}\left(p(t)+\gamma\left|x_{1}\right|\right)
$$

is $[0,1] \times \mathbb{R} \times \mathbb{R}$ and this is why it is more convenient for us to study an auxiliary equation (3.31).

We define a function $\theta: \mathbb{R} \times \mathbb{R} \rightarrow \mathbb{R}_{+}$by formula $(3.10)$ and define the function $d^{\theta}$ in the space $\mathbb{S}$.

For an arbitrary function $v \in \mathbb{S}$ we define functions $g_{1}^{[v]}, g_{2}^{[v]}:[0,1] \times \mathbb{R} \rightarrow \mathbb{R}$ as

$$
g_{1}^{[v]}(t, x)=x^{2}(p(t)+\gamma|v(h(t))|), \quad g_{2}^{[v]}(t, x)=v^{2}(t)(p(t)+\gamma|x|) .
$$

We let $\alpha=1, \beta=1 / 2$ and $x_{0}(t) \equiv 0$ on $[0,1]$. The value $R$ calculated by formula (3.28) coincides with (3.30). As it has been shown in Example 1, the function $g_{1}^{[v]}$ satisfies condition (3.8), in which $\Omega(t) \equiv \mathbb{R}$, and $x, y \in \mathbb{S}$ are arbitrary functions including the case $x \in B_{\mathbb{S}^{\theta_{0}}}\left(x_{0}, R\right)$ and $y=\widehat{y}$ is a given right hand side of equation (3.29).

According Example 3, the function $g_{2}(t, x)=p(t)+\gamma|x|$ satisfies condition (3.19) with the coefficient $\gamma$ for all $x, u \in \mathbb{R}$. It follows from inequalities (3.12), (3.13) that for each $v \in B_{\mathbb{S}_{0}}\left(x_{0}, R\right)$ the function $g_{2}^{[v]}$ satisfies condition $(3.19)$ with the coefficient

$$
\max \left\{\gamma R^{2}, \gamma R\right\} \leqslant \frac{1}{2}=\beta
$$

According Theorem 3.1, equation (3.31), and hence, equation (3.29), possesses a solution $x \in \mathbb{S}_{+}$such that $x(t) \leqslant R$ a.e. on $[0,1]$.

\section{Cauchy problem for an implicit differential EQUation}

In the space $\mathbb{S}=\mathbb{S}([0, \tau], \mathbb{R})$, we select a subspace $\mathbb{L}=\mathbb{L}([0, \tau], \mathbb{R})$ of Lebesgue summable functions. This space with a distance $d^{\theta}$ defined by formula $(3.2)$ is denoted by $\mathbb{L}^{\theta}$. The space $\mathbb{L}^{\theta_{0}}$ is complete. We note that for each $x \in \mathbb{L}, r \in \mathbb{R}_{+}$we have $B_{\mathbb{L}^{\theta_{0}}}(x, r)=B_{\mathbb{S}^{\theta_{0}}}(x, r)$. We denote by $\mathbb{A} \mathbb{C}=\mathbb{A} \mathbb{C}([0, \tau], \mathbb{R})$ the space of absolutely continuous functions $x:[0, \tau] \rightarrow \mathbb{R}$ having a derivative $\dot{x} \in \mathbb{L}$ a.e. on $[0, \tau]$.

Let a function $\widehat{y}: \mathbb{R}_{+} \rightarrow \mathbb{R}$ be measurable, the function $f: \mathbb{R}_{+} \times \mathbb{R} \times \mathbb{R} \rightarrow \mathbb{R}$ be measurable with respect to the first variable and is jointly continuous with respect to the second and third variables. We consider an implicit differential equation

$$
f(t, x(t), \dot{x}(t))=\widehat{y}(t), \quad t \geqslant 0 .
$$


Let $\tau>0$. A solution to equation (4.1) defined on $[0, \tau]$ is a function $x \in \mathbb{A}([0, \tau], \mathbb{R})$ satisfying this equation for a.e. $t \in[0, \tau]$. We are going to obtain conditions on existence of solution $x \in \mathbb{A} \mathbb{C}([0, \tau], \mathbb{R})$ to equation (4.1) satisfying the initial condition

$$
x(0)=A
$$

for a given $A \in \mathbb{R}$.

For arbitrary functions $v \in \mathbb{A} \mathbb{C}([0, \tau], \mathbb{R})$ and $w \in \mathbb{L}([0, \tau], \mathbb{R})$ we define the functions $g_{1}^{[v]}, g_{2}^{[w]}$ : $[0, \tau] \times \mathbb{R} \rightarrow \mathbb{R}$ by the relations

$$
g_{1}^{[v]}(t, x)=f(t, v(t), x), \quad g_{2}^{[w]}(t, x)=f(t, x, w(t)), \quad t \in[0, \tau], \quad x \in \mathbb{R} .
$$

Theorem 4.1. Suppose that we are given numbers $\alpha>0, \beta \geqslant 0, \tau>0$ such that $\beta \tau<\alpha$ and a function $x_{0} \in \mathbb{A} \mathbb{C}([0, \tau], \mathbb{R})$ satisfying condition (4.2). Let

$$
R:=\frac{1}{\alpha-\beta \tau} \text { vrai } \sup _{t \in[0, \tau]} \theta\left(f\left(t, x_{0}(t), \dot{x}_{0}(t)\right), \widehat{y}(t)\right)<\infty .
$$

We define multi-valued mappings $V, \dot{V}:[0, \tau] \rightrightarrows \mathbb{R}$ by the relations

$$
V(t)=B_{\mathbb{R}}\left(x_{0}(t), R t\right), \quad \dot{V}(t)=B_{\mathbb{R}}\left(\dot{x_{0}}(t), R\right), \quad t \in[0, \tau] .
$$

Assume that for each absolutely continuous function $v \in \operatorname{Sel}(V)$, the function $g_{1}^{[v]}$ satisfies condition (3.8) for $y=\widehat{y}$, all $x \in \operatorname{Sel}(\dot{V}), \Omega(t) \equiv \mathbb{R}$ and that for each $w \in \operatorname{Sel}(\dot{V})$ the function $g_{2}^{[w]}$ satisfies condition (3.17) for $y=\widehat{y}$, all absolutely continuous functions $x \in \operatorname{Sel}(V)$ and $\Omega=V$. Then there exists a solution $x$ to problem 4.1, 4.2 defined on $[0, \tau]$ such that $\dot{x} \in B_{\mathbb{L}^{\theta_{0}}}\left(\dot{x}_{0}, R\right)$.

Proof. We write problem (4.1), 4.2) as the equation

$$
f\left(t, A+\int_{0}^{t} u(s) d s, u(t)\right)=\widehat{y}(t), \quad t \in[0, \tau],
$$

for an unknown function $u=\dot{x} \in \mathbb{L}([0, \tau])$. We define a mapping

$$
F: \mathbb{L}^{\theta_{0}} \times \mathbb{L}^{\theta_{0}} \rightarrow \mathbb{S}^{\theta}, \quad(F(u, z))(t)=f\left(t, A+\int_{0}^{t} z(s) d s, u(t)\right), \quad t \in[0, \tau],
$$

and a mapping $G: \mathbb{L}^{\theta_{0}} \rightarrow \mathbb{S}^{\theta}, G(u)=F(u, u)$. Under such definition of the mapping $G$, equation (4.5) becomes (2.1) and its solvability can be proved on the base of Theorem 2.1; we are going to check the assumptions of this theorem.

We begin with proving the closedness of the mappings $F, G$. Let for some $\left\{u_{i}\right\},\left\{z_{i}\right\} \subset \mathbb{L}^{\theta_{0}}$, $u, z \in \mathbb{L}^{\theta_{0}}$ and $y \in \mathbb{S}^{\theta}$ we have $\rho\left(u_{i}, u\right) \rightarrow 0, \rho\left(z_{i}, z\right) \rightarrow 0$ and $d^{\theta}\left(F\left(u_{i}, z_{i}\right), y\right) \rightarrow 0$. The latter relation implies the convergence $\left(F\left(u_{i}, z_{i}\right)\right)(t) \rightarrow y(t)$ for a.e. $t \in[0, \tau]$, see the proof of Proposition 1. By the second relation we obtain $\int_{0}^{t} z_{i}(s) d s \rightarrow \int_{0}^{t} z(s) d s$ for a.e. $t \in[0, \tau]$. By the continuity of the function $f(t, \cdot, \cdot)$, for a.e. $t \in[0, \tau]$ we have $\left(F\left(u_{i}, z_{i}\right)\right)(t) \rightarrow(F(u, z))(t)$. And since $\left(F\left(u_{i}, z_{i}\right)\right)(t) \rightarrow y(t)$, we obtain $(F(u, z))(t)=y(t), t \in[0, \tau]$. Thus, we have proved that the mapping $F$ is closed and respectively, the mapping $G$ is also closed.

For an arbitrary function $w \in \operatorname{Sel}(\dot{V})$, the operator $F(\cdot, w): \mathbb{L}^{\theta_{0}} \rightarrow \mathbb{S}^{\theta}$ is the Nemytskii operator $N_{g_{1}^{[v]}}$ generated by the function $g_{1}^{[v]}$, where $v(t)=A+\int_{0}^{t} w(s) d s$. It is obvious that $v \in \operatorname{Sel}(V)$ and $v \in \mathbb{A} \mathbb{C}([0, \tau], \mathbb{R})$. According our assumptions, the operator $N_{g_{1}^{[v]}}$ satisfies the assumptions of Proposition 2 for $y=\widehat{y}$, each $x \in \operatorname{Sel}(\dot{V})$ and $\Omega(t) \equiv \mathbb{R}$. According Proposition 2 , the embedding $\operatorname{Sel}(\dot{V}) \times\{\widehat{y}\} \subset \operatorname{Cov}_{\alpha}[F(\cdot, w) ; \mathbb{S}]$ holds and therefore, $(w, \widehat{y}) \in \operatorname{Cov}_{\alpha}[F(\cdot, w) ; \mathbb{S}]$.

Now we consider the operator $F(w, \cdot): \mathbb{L}^{\theta_{0}} \rightarrow \mathbb{S}^{\theta}$, where $w$ is an arbitrary measurable section of the multi-valued mapping $\dot{V}$. The operator $F(w, \cdot)$ is a composition of the integral operator $K: \mathbb{L}^{\theta_{0}} \rightarrow \mathbb{L}^{\theta_{0}}$ defined by the formula $(K z)(t)=\int_{0}^{t} z(s) d s$ and the Nemytskii operator $N_{g_{2}^{[w]}}$ : 
$\mathbb{L}^{\theta_{0}} \rightarrow \mathbb{S}^{\theta}$ generated by the function $g_{2}^{[w]}$. The operator $K$ with the coefficient $\tau$ on the set $\operatorname{Sel}(\dot{V})$ and $K(\operatorname{Sel}(\dot{V})) \subset \mathbb{A} \mathbb{C} \cap \operatorname{Sel}(V)$. The function $g_{2}^{[w]}$ satisfies the assumptions of Proposition 3 with $y=\widehat{y}$, each $x \in \mathbb{A} \mathbb{C} \cap \operatorname{Sel}(V)$ and $\Omega=V$. Hence, by Proposition 3 , for each $x \in \mathbb{A} \cap \operatorname{Sel}(V)$ we have $(x, \widehat{y}) \in \operatorname{Lip}_{\beta}\left[N_{g_{2}^{[w]}} ; \operatorname{Sel}(V)\right]$. Therefore, $(z, \widehat{y}) \in \operatorname{Lip}_{\beta \tau}\left[N_{g_{2}^{[w]}} K ; \operatorname{Sel}(\dot{V})\right]$ for each $z \in \operatorname{Sel}(\dot{V})$. Thus, $\operatorname{Sel}(\dot{V}) \times \widehat{y} \subset \operatorname{Lip}_{\beta \tau}\left[N_{g_{2}^{[w]}} K ; \operatorname{Sel}(\dot{V})\right]$, and hence, $(w, \widehat{y}) \in \operatorname{Lip}_{\beta \tau}\left[N_{g_{2}^{[w]}} K ; \operatorname{Sel}(\dot{V})\right]$.

Hence, equation (4.5) satisfies the assumptions of Theorem 2.1 and this is why this equation possesses a solution $\widehat{u} \in \operatorname{Sel}(\dot{V})=B_{\mathbb{L}^{\theta_{0}}}\left(\dot{x}_{0}, R\right)$. Hence, there exists a solution $x$ to problem 4.1), 4.2 defined on $[0, \tau]$ such that $\dot{x} \in B_{\mathbb{L}^{\theta_{0}}}\left(\dot{x}_{0}, R\right)$.

Example 7. Suppose that we are given measurable non-negative functions $p, \widehat{y}: \mathbb{R}_{+} \rightarrow \mathbb{R}_{+}$ and a number $\gamma \geqslant 0$. We assume that $p(t) \geqslant 1$ for a.e. $t \geqslant 0$. We consider a differential equation

$$
\dot{x}^{2}(t)(p(t)+\gamma|x(t)|)=\widehat{y}(t), \quad t \geqslant 0 .
$$

We let

$$
R:=2 \text { vrai } \sup _{t \in[0,1]} \widehat{y}(t) .
$$

We are going to show that for each $\tau>0$ such that

$$
2 \gamma R \tau<1, \quad 2 \gamma R^{2} \tau<1,
$$

there exists a solution $x$ to equation (4.6) defined on $[0, \tau]$ obeying initial condition $x(0)=0$ such that $|\dot{x}(t)| \leqslant R$ for a.e. $t \in[0, \tau]$.

We fix and arbitrary $\tau>0$ satisfying inequalities (4.8). We define a function $\theta: \mathbb{R} \times \mathbb{R} \rightarrow \mathbb{R}_{+}$ by formula $\left(3.10\right.$ and an appropriate distance $d^{\theta}$ in the space $\mathbb{S}$. For arbitrary functions $v \in \mathbb{A} \mathbb{C}$ and $w \in \mathbb{L}$ we define functions $g_{1}^{[v]}, g_{2}^{[w]}:[0, \tau] \times \mathbb{R} \rightarrow \mathbb{R}$ by the relations

$$
g_{1}^{[v]}(t, x)=x^{2}(p(t)+\gamma|v(t)|), \quad g_{2}^{[w]}(t, x)=w^{2}(t)(p(t)+\gamma|x|), \quad t \in[0, \tau], \quad x \in \mathbb{R} .
$$

We let $x_{0}(t) \equiv 0$ on $[0, \tau]$. We define multi-valued mappings $V, \dot{V}:[0, \tau] \rightrightarrows \mathbb{R}$ by formulae (4.4).

For each function $v \in \mathbb{A} \mathbb{C}$, including $v \in \operatorname{Sel}(V) \cap \mathbb{A}$, the function $g_{1}^{[v]}$ satisfies condition (3.8) with the coefficient $\alpha=1$ for each $x, y \in S$, including $y=\widehat{y}$ and $x \in \operatorname{Sel}(\dot{V})$, and $\Omega(t) \equiv \mathbb{R}$, see Example 6. For each $w \in \operatorname{Sel}(\dot{V})$, the function $g_{2}^{[w]}$ satisfies Condition 3.17) with the coefficient $\beta=\max \left\{\gamma R, \gamma R^{2}\right\}$ for $y=\widehat{y}$, all absolutely continuous functions $x \in \operatorname{Sel}(V)$ and $\Omega=V$, see Example 6.

By inequalities (4.8) we have $\beta \leqslant(2 \tau)^{-1}$. Thus, $\alpha-\beta \tau \geqslant 2^{-1}$. Therefore, the value $R$ calculated by formula (4.7) does not exceed value (4.3). In accordance with Theorem 4.1, there exists a solution $x$ to equation (4.6) defined on $[0, \tau]$ such that $x(0)=0$ and $|\dot{x}(t)| \leqslant R$ a.e. on $[0, \tau]$.

\section{BIBLIOGRAPHY}

1. A.V. Arutyunov, E.S. Zhukovskiy, S.E. Zhukovskiy. Covering mappings and well-posedness of nonlinear Volterra equations // Nonl. Anal. Theory, Methods and Applications. 75:3, 1026-1044 (2012).

2. E.R. Avakov, A.V. Arutyunov, E.S. Zhukovskii. Covering mappings and their applications to differential equations unsolved for the derivative // Differ. Uravn. 45:5, 613-634 (2009). [Diff. Equat. 45:5, 627-649 (2009).]

3. A.V. Arutyunov, E.S. Zhukovskii, S.E. Zhukovskii. On the well-posedness of differential equations unsolved for the derivative // Differ. Uravn. 47:11, 1523-1537 (2011). [Diff. Equat. 47:11, 15411555 (2011).]

4. E.S. Zhukovskii, E.A. Pluzhnikova. Covering mappings in a product of metric spaces and boundary value problems for differential equations unsolved for the derivative // Differ. Uravn. 49:4, 439-455 (2013). [Diff. Equat. 49:4, 420-436 (2013).] 
5. E.S. Zhukovskii, E.A. Pluzhnikova. On controlling objects whose motion is defined by implicit nonlinear differential equations // Avtom. Telemekh. 1, 31-56 (2015). [Autom. Remote Control. 1, 24-43 (2015).]

6. A.V. Arutyunov, S.E. Zhukovskii. Coincidence points of mappings in vector metric spaces with applications to differential equations and control systems // Differ. Uravn. 53:11, 1473-1481 (2017). [Diff. Equat. 53:11, 1440-1448 (2017).]

7. A.V. Arutyunov, A.V. Greshnov. Theory of $\left(q_{1}, q_{2}\right)$-quasimetric spaces and coincidence points // Dokl. RAN. 469:5, 527-531 (2016). [Dokl. Math. 94:1, 434-437 (2016).]

8. W. Merchela. On Arutyunov theorem of coincidence point for two mapping in metric spaces // Tambov Univ. Rep. Ser. Natur. Techn. Sci. 23:121, 65-73 (2018). (in Russian).

9. E.S. Zhukovskiy, W. Merchela. On the continuous dependence on the parameter of the set of solutions of the operator equation // Izv. Inst. Mat. Inform. Udmurt. Gos. Univ. 54, 27-37 (2019). (in Russian).

10. S. Benarab, E.S. Zhukovskii, W. Merchela. Theorems on perturbations of covering mappings in spaces with a distance and in spaces with a binary relation // Trudy Inst. Matem. Mekh. UrO RAN. 25:4, 52-63 (2019). (in Russian).

11. A.V. Arutyunov. Covering mappings in metric spaces and fixed points // Dokl. RAN. 416:2, 151-155 (2007). [Dokl. Math. 76:2, 665-668 (2007).]

12. Yu.G. Borisovich, B.D. Gel'man, A.D. Myshkis, V.V. Obukhovskii. Introduction to the theory of multi-valued mappings and differential inclusions. Librokom, Moscow (2011). (in Russian).

13. N. Dunford, J.T. Schwartz. Linear operators. I. General theory. Interscience Publ., New York (1958).

14. N. Azbelev, V. Maksimov, L. Rakhmatullina. Introduction to the theory of linear functional differential equations. Nauka, Moscow (1991). [World Federation Publishers Comp., Atlanta (1995).]

Evgeny Semenovich Zhukovskiy,

Derzhavin Tambov State University,

Internatsionalnya str. 33,

392000, Tambov, Russia

Trapeznikov Institute of Control Sciences,

Profsoyuznaya str. 65,

117997, Moscow, Russia

E-mail: zukovskys@mail.ru

Wassim Merchela,

Derzhavin Tambov State University,

Internatsionalnya str. 33,

392000, Tambov, Russia

Laboratoire des Mathématiques Appliquées et Modélisation,

Université 8 Mai 1945 Guelma,

B.P. 401,

24000, Guelma, Algeria

E-mail: merchela.wassim@gmail.com 